Théologiques

Théologiques

\title{
Le champ de l'herméneutique
}

Trajectoires et carrefours

\section{Jean-Paul Resweber}

Volume 10, numéro 2, automne 2002

Théologie et psychanalyse. Que dit l'une au sujet de l'autre?

URI : https://id.erudit.org/iderudit/008883ar

DOI : https://doi.org/10.7202/008883ar

Aller au sommaire du numéro

\section{Éditeur(s)}

Faculté de théologie de l'Université de Montréal

ISSN

1188-7109 (imprimé)

1492-1413 (numérique)

Découvrir la revue

Citer cet article

Resweber, J.-P. (2002). Le champ de l'herméneutique : trajectoires et carrefours. Théologiques, 10(2), 55-78. https://doi.org/10.7202/008883ar
Résumé de l'article

Du point de vue de la démarche philosophique, l'a. présente trois trajectoires herméneutiques distinctes, pour en déterminer les points d'articulation et les convergences: la phénoménologie (Husserl, Heidegger, Gadamer, Ricoeur), la psychanalyse (Freud, Lacan), la théologie (Bultmann, Derrida). Quelques " lieux communs » de l'herméneutique apparaissent alors: traversée et quête renouvelée d'une compréhension où il n'y a point de vérité absolue, écriture codée du texte ou des inscriptions vécues et écriture parlée du dialogue philosophique ou analytique, tension entre le littéral et le figuré,

compréhension perçue comme traduction et compréhension perçue comme écoute, partage sur la question du fondement, rapport privilégié entre le récit et la configuration temporelle de la vérité, et précarité du sujet-interprète douloureusement éprouvée par le sujet de la théologie. d'utilisation que vous pouvez consulter en ligne.

https://apropos.erudit.org/fr/usagers/politique-dutilisation/ 


\title{
Le champ de l'herméneutique Trajectoires et carrefours
}

\author{
Jean-Paul Resweber \\ Institut de philosophie \\ Université de Metz
}

Ce que l'on appelle herméneutique recouvre trois démarches intellectuelles distinctes. La première, de type méthodologique, dont l'exégèse biblique fournit le modèle, définit les règles de l'interprétation des textes. Elle convoque des disciplines variées, allant de la philologie aux sciences humaines en passant par la grammaire, la logique et la rhétorique, afin de restituer le sens du texte qui est supposé exprimer les intentions de l'auteur. Friedrich Schleiermacher pose les bases d'une telle pratique qui porte aussi bien sur la lecture du Nouveau Testament ou la traduction de Platon que sur les textes des présocratiques. Outre l'analyse grammaticale, il recourt à une analyse technique qui, centrée sur le style, met en évidence l'usage créatif que l'auteur fait de la langue ${ }^{1}$. Mais cet art de comprendre reste trop psychologique, tributaire qu'il est d'une théorie du «moi » parlant. C’est Wilhelm Dilthey qui donne à la méthode une assise plus objective: la compréhension des "monuments écrits» prend sans doute appui sur les données de l'histoire, mais elle considère celles-ci comme autant de témoignages de $v^{2} e^{2}$. En orientant l'herméneutique vers le déchiffrement historique du vécu, il dépasse aussi bien le subjectivisme de Schleiermacher que le positivisme de l'historicisme ambiant. Il transforme ainsi la méthode en une démarche épistémologique qui s'applique à découvrir les critères fondamentaux de l'interprétation. En s'attelant à

1. F. Schleiermacher, Herméneutique / trad. par C. Berner, Paris, Cerf, 1987 (allemand 1805-1833).

2. W. Dilthey, "Origines de l'herméneutique ", dans Le Monde de l'esprit / trad. par M. Rémy, t. 1, Paris, Aubier, 1947 (allemand 1900), p. 322. 
ce travail de légitimation que poursuivront Martin Heidegger et Paul Ricœur, Dilthey élargit, d'une part, le champ de l'herméneutique, en lui assignant pour objet non seulement le texte, mais aussi les multiples secteurs du monde vécu et, d'autre part, il enrichit les outils de l'interprétation, en faisant appel aux nouvelles sciences humaines. On peut, enfin, entendre par herméneutique, comme c'est le cas de la phénoménologie, de la psychanalyse et de la théologie, une démarche philosophique, qui englobe les deux démarches précédentes. C'est en nous situant dans cette dernière perspective que nous suivrons les trois trajectoires herméneutiques de la phénoménologie, de la psychanalyse et de la théologie et que nous dégagerons les lieux communs ou les carrefours, où elles se recoupent.

\section{Trajectoires}

\subsection{L’héritage de la phénoménologie \\ 1.1.1. Husserl}

On peut dire que la démarche de la phénoménologie s'inscrit dans la logique herméneutique de la réflexion philosophique. L'attention aux seuls phénomènes ou le retour aux choses fait, en effet, table rase des systèmes qui représentent autant d'interprétations figées et bloquées. Husserl ne cherche pas à légitimer le cogito, mais à en ressaisir la signification intersubjective, morale et ontologique qui, quoique non attestée explicitement par le texte cartésien, n'en est pas moins présente. On le voit, la tâche herméneutique exige que l'on questionne, en repartant à zéro, le phénomène, qu'on le débarrasse des thèses et hypothèses qui en maquillent la réalité. Mais il n'y a pas que les systèmes qui travestissent l'essence de la chose: il y a aussi tous les préjugés de l'opinion commune. C'est pourquoi la réduction philosophique, qui s'en prend aux idées reçues, est inséparable de la réduction phénoménologique, qui soustrait le phénomène aux projections de la conscience spontanée ou naturelle.

Un tel geste réduit les erreurs et les préjugés à des malentendus, qui bloquent l'interprétation. L'analyse du phénomène passe par la réduction transcendantale. Elle s'emploie, d'une part, à élaguer du phénomène les variations qui l'affectent, pour ne retenir que son être essentiel, son « eidos » et, d'autre part, à rattacher l'essence ainsi découverte à la visée de la conscience qui le fonde en le posant. Ce double jeu corrélatif de la 
réduction eidétique et de la réduction transcendantale illustre, en réalité, les deux moments de tout travail interprétatif: celui du décollage des stratifications qui composent le profil historique du phénomène et celui du retour aux postures originelles de la conscience dont le regard constitue l'objet. Cette logique régressive et réductrice est inséparable d'une logique progressive. Allant du monde vécu à la conscience instituante, l'interprétation revient de la conscience vers le monde, car la remontée au fondement n'a d'autre but que de permettre à la conscience de réhabiter le monde, mais autrement, avec recul, distance et critique. La traversée de la genèse se trouve finalisée par les repositionnements plus ajustés de la conscience au monde. Ce que Husserl appelle la constitution du monde a pour but de rectifier "la thèse du monde » initialement posée dans le déni herméneutique de la conscience de soi. Ainsi, on le voit, ce parcours se trouve-t-il, en définitive, fondé sur une éthique du soi, se découvrant sans cesse comme autre. C'est cette détermination qui, destituant la conscience de son statut transcendantal, autorise à la considérer comme une conscience herméneutique engagée dans le monde qu'elle vise.

Pour emprunter le langage de Husserl, on peut dire que l'herméneutique consubstantielle à la phénoménologie va d'une prise de conscience noématique à une prise de conscience noétique et, inversement, d'une prise de conscience noétique à une prise de conscience noématique. Il existe, en effet, deux plans où s'exerce la visée de la conscience: celui de l'intentionnalité thématique et opérationnelle, qui prend conscience du regard actuel qu'elle pose sur l'objet, et celui de l'intentionnalité nonthématique et opérante (die fungierende Intentionalität), qui échappe à toute réflexivité. Or, c'est en se ressourçant à la conscience irréfléchie que la conscience réfléchie ressaisit le sens factuel des "vécus ". L'intentionnalité opérante est, par conséquent, le préalable nécessaire à tout geste d'élucidation des données de conscience, puisqu'elle circonscrit le champ où les objets se phénoménalisent, en étant marqués au coin des synthèses catégoriales. Mais ces opérations préalables, qui ne sont pas conscientes, s'organisent en deux strates de sens superposés: celle des strates archaïques issues des visées originelles de la conscience et celle des strates issues des dépôts successifs de sens thématiquement visés. Ces dernières, chassées par les intentionnalités explicites, retombent en deçà des visées actuelles de la conscience, pour épaissir les sédimentations passives de l'intentionnalité primaire et anonyme de la conscience. C'est à cette inscience qui, sans cesse renforcée, résiste à l'interprétation que la conscience se 
ressource pour reconstituer ses vécus et se produire elle-même comme autre ${ }^{3}$.

\subsubsection{Heidegger}

Heidegger se propose de greffer la visée herméneutique de la phénoménologie sur l'ontologie. On se tromperait cependant à considérer que le tournant ontologique pervertit la démarche herméneutique, au point d'assimiler la philosophie de Heidegger à une phénoménologie sans herméneutique. Bien au contraire. Mais le projet de Heidegger est complexe. Il cherche à opérer le croisement d'une herméneutique de la métaphysique et d'une herméneutique de l'existence humaine, au lieu même de la question de l'être. Les thèmes de la destruction et de la déconstruction, ceux du retour au fondement de la métaphysique témoignent avec force de la volonté d'interpréter l'écriture même de la métaphysique comme une rhapsodie de l'oubli de la question de l'être. Parallèlement, la problématique philosophique de l'existence humaine se réalise dans une herméneutique du Dasein qui libère la question de l'être de sa captation et de sa capture par la raison subjective. On le voit, le discours de la métaphysique éclôt dans le discours d'une subjectivité qui s'affirme comme étant la raison ultime de l'être. C'est bien à déconstruire la subjectivité que s'emploie Heidegger, aussi bien celle qui s'inscrit comme motif de la métaphysique que celle qui se cache dans la léthargie anthropologique de la banalité quotidienne. L'herméneutique de Heidegger allie la dénonciation au déchiffrage dans un même processus de déconstruction. L'enjeu est d'importance, car c'est l'illusion de l'absoluité de la subjectivité qui est la cause de l'errance et des incohérences de notre modernité.

Que la subjectivité se décline comme conscience, cogito ou intersubjectivité, qu'elle plonge dans l'anonymat du bavardage ou de la curiosité, qu'elle s'excède dans la figure de la nation, du peuple ou de l'État, elle témoigne, de par sa position même, de l'oubli fatal de l'être. Or, l'être est le lieu d'une question qu'il faut bien tirer de l'oubli, pour enfin interpréter la constitution même de cet être singulier qu'est l'être humain. Accepter cette question, c'est prendre du recul par rapport à toutes les formes

3. Voir sur ce sujet: M. Henry, «Ricœur et Freud. Entre psychanalyse et phénoménologie ", dans J. Greisch et R. Kearney, dir., Paul Ricour. Les métamorphoses de la raison herméneutique, Paris, Cerf, 1991, p. 127. 
de la subjectivité. C'est aussi envisager l'homme autrement: le considérer comme inscrit dans le champ de l'être-au-monde, qui est la raison de sa transcendance, de sa finitude. Tel est le sens du tournant heideggérien (Kehre), qui est moins l'expression d'un pari ou d'un défi que celle d'une volonté de penser autrement, moins sur le mode d'une reprise réflexive que sur celui d'une répétition herméneutique. C'est du lieu de l'être, et non du lieu de la subjectivité, que l'essence de l'homme peut être conçue, c'est-à-dire élucidée, explicitée, interprétée. Or, un tel tournant, dont témoigne toute décision philosophique et qui exprime ici la volonté de fonder la pensée sur l'herméneutique, comporte deux moments: celui qui, présidant au saut (Satz), de la raison subjective vers l'être, nous introduit à l'herméneutique du Dasein et celui qui, prenant acte de la négativité que le temps institue au cœur de l'être, inaugure l'herméneutique poétique de la vérité de l'être. Heidegger propose de lier ces deux moments : celui de l'herméneutique de l'existence qui s'accomplit dans la compréhension de l'être finalisé par la mort et celui de la compréhension de l'être ainsi éprouvée et qui échoue dans la nuit du temps. Il entend ainsi définir moins une trajectoire particulière de l'herméneutique que circonscrire l'espace même de toute herméneutique possible: celui qui, au plan de l'écriture, bute sur la barrière de la mort et celui qui, au plan de l'être, bute sur la barrière du temps. C'est au langage du poète que Heidegger confie le soin de faire entendre l'écho de la mort et du temps: du temps de la mort, de la mort comme sursis. C'est cet écho que l'herméneutique interprète, en lui prêtant la voix du discours.

\subsubsection{Gadamer}

L'herméneutique de Hans-Georg Gadamer revendique l'analyse de la finitude qui fonde la légitimité de l'herméneutique existentiale de Heidegger $^{4}$. Ainsi, sur bien des points essentiels, l'auteur de Vérité et méthode prolonge les vues développées par celui de L'Être et le Temps, notamment

4. H.G. Gadamer, Vérité et méthode. Les grandes lignes d'une herméneutique philosophique / édition intégrale révisée et complétée / trad. par P. Fruchon, J. Grondin et G. Merlio, Paris, Seuil, 1996 (1976; allemand 1960); L'art de comprendre / trad. par M. Simon, t. 1 (Herméneutique et tradition philosophique) et t. 2 (Herméneutique et champ de l'expérience humaine), Paris, Aubier-Montaigne, 1982 et 1991. 
celles qui portent sur les notions de compréhension, de discursivité et de vérité. Il articule, de façon originale, le modèle de la compréhension à l'expérience de l'art et de la rhétorique. Il considère, en effet, qu'il n'y a point de sensibilité esthétique qui ne suppose l'engagement actif de l'interprète dans la contemplation de l'œuvre d'art. C'est à l'occasion de cette démarche spécifique que, selon Gadamer, se produit l'illumination de la beauté, qui accompagne la présentation de l'œuvre d'art comme telle. La compréhension, en effet, actualise et accomplit une précompréhension, tissée d'attentes et de préjugés qui se trouvent tantôt légitimés, tantôt récusés. Elle surgit, paradoxalement, de l'application du message à la situation de l'interprète. C'est dire que la réception du message fait structurellement partie de la révélation de la beauté ou de la vérité. L'interprétation est un événement qui se produit à la croisée de l'appel de l'œuvre et de la réponse que lui fait l'interprète. La rhétorique, quant à elle, confirme ce modèle et permet d'en préciser les limites. La vérité que les figures du langage éveillent chez celui auquel elles s'adressent n'est pas, en effet, de l'ordre de l'illumination ou de la fulgurance, comme c'est le cas dans l'expérience esthétique: elle est de l'ordre de la vraisemblance, car elle «clarifie» notre situation, met en relief notre monde environnant (Lebenswelt) et, ce faisant, déploie l'horizon du sens qui suffit à orienter notre praxis.

L'herméneutique de Gadamer accorde une fonction positive aux préjugés hérités de la tradition dans la mise en œuvre de la compréhension. Ces derniers la paralysent, la préparent ou l'orientent; ils se trouvent tantôt éradiqués, tantôt confirmés, tantôt remaniés. Mais quel est le processus qui préside à ce travail de correction et de sélection? Gadamer propose deux réponses à cette question. C'est la distance temporelle ou le recul du temps, d'abord, qui intervient, pour mettre les préjugés à l'épreuve de la falsification. Mais il en appelle aussi à la performativité du dialogue que l'interprète noue avec le monde, avec le texte ou avec autrui. La résistance de l'objet, de l'œuvre ou de la question l'amène, dès lors, à retenir comme légitimes les préjugés qui servent de relais à la discussion et fournissent la matrice d'une juste compréhension. On ne saurait pour autant prendre en compte ce jeu de «questions-réponses ", qui est au cœur de la démarche herméneutique de Gadamer, sans faire appel à une conception spécifique du langage et de la vérité historique. 
La compréhension du sens, qui fait appel au tact et à l'empathie et relève de l'induction " $\operatorname{artistique~}^{5}$ ", se passe, à la limite, de la vérification logique sanctionnée par la méthode. Car elle se réalise toujours dans l'espace dialogique des questions et des réponses. Toute proposition est, en effet, une réponse individuelle ou collective à une question qu'il faut déchiffrer après-coup. Elle cristallise un vouloir-dire qui déborde l'énoncé et dans lequel la conception instrumentale du langage veut l'enfermer. Le discours extérieur est l'expression d'un discours intérieur, d'une «verbum cordis", selon l'expression que Gadamer emprunte à saint Augustin. Il énonce ce qui s'annonce d'un sens qui demeure toujours en retrait et que seule une herméneutique du cœur peut délivrer. Il reflète en miroir, en la reconfigurant, la virtualité langagière (Sprachlichkeit) qui constitue l'arrière-fond dialogique de tout discours. Voilà pourquoi l'herméneutique accomplit une entente "spéculative» procédant d'un dialogue engagé non seulement sur la base de ce que l'auteur et l'interlocuteur ont proféré, mais aussi sur la base de ce qu'ils ont voulu dire, mais n'ont pu dire. C'est bien sûr à partir des énoncés entendus et des énoncés proférés que ce travail d'interprétation peut se réaliser. On le voit, la conception langagière de H.G. Gadamer recoupe celle de la pragmatique linguistique qui considère les figures rhétoriques comme autant d'expressions du vouloirdire. C'est à ressaisir, dans le dialogue, l'intentionnalité linguistique que l'herméneutique fait advenir le sens à la compréhension.

Selon Gadamer, la compréhension est historique, en un sens radical. Il existe, en effet, une histoire de l'efficience (Wirkungsgeschichte) qui conditionne toute prise de conscience de la vérité. Or, cette efficience, qui résulte d'un véritable travail de l'histoire comme telle, pose celle-ci en référence transcendantale. Il existe, pour Gadamer comme pour Heidegger, un destin de l'être. Cette idée-force souligne, à vrai dire, le rôle fondamental que joue la tradition dans la compréhension de la vérité. Mais H.G. Gadamer n'entend pas pour autant voir dans la tradition l'imposition d'un sens de l'histoire qui ne correspondrait ni aux capacités ni aux attentes de l'intelligence humaine. S'il existe une rationalité de l'histoire, celle-ci ne devient effective que dans et par la conscience réfléchie qui se

5. H. von Helmholtz distingue l'induction logique, qui explique un phénomène en le subsumant sous un concept qui fait loi et l'induction artistique, qui comprend le phénomène par empathie, tact ou intuition. Voir H. von Helmholtz, Vorträge und Reden, Braunschweig, Verlag F. Vieweg und Sohn, 1884, p. 119s. 
l'approprie. La vérité est ainsi un avènement de sens qui suppose l'ouverture de l'homme à la tradition, mais elle est aussi la façon dont l'homme mesure cette ouverture, pour l'appliquer à sa situation. Ainsi, selon H.G. Gadamer, elle émerge du travail conjugué de la tradition qui la devance et de l'appropriation qu'elle en effectue. Voilà pourquoi elle ne saurait déboucher sur une certitude absolue. Tout au plus se réalise-t-elle dans une compréhension "autre» (Andersverstehen), dans la découverte de nouveautés qui sont autant de chances de connaissances (Erkenntnischancen), autant d'horizons prometteurs qui surgissent de la reprise des horizons du passé à la lumière des horizons de l'avenir.

\subsubsection{Ricœur}

À l'instar de l'herméneutique de Gadamer, l'herméneutique de Ricœur résulte d'une greffe épistémologique effectuée sur le tronc de la phénoménologie. Elle comporte trois traits principaux, de l'aveu même de l'auteur ${ }^{6}$. L'un relève de la philosophie réflexive, l'autre renvoie à la mouvance husserlienne, le dernier, enfin, développe une variante herméneutique de la phénoménologie. Nous n'expliciterons pas le premier volet, qui regroupe un ensemble de recherches sur la symbolique du mal et qui se réclame de la philosophie réflexive: le travail de l'interprétation consiste ici à rapporter le développement de la pensée à l'acte originaire qui la fonde ${ }^{7}$. Nous n'insisterons pas non plus sur le deuxième volet, qui déplace l'herméneutique du pôle de la symbolique du mal vers celui de la psychanalyse freudienne et du conflit des interprétations ${ }^{8}$. Nous soulignons seulement l'originalité du troisième volet, qui nous renvoie à l'expérience de la temporalité qui, inhérente au langage et à la réalisation de soi comme autre, trouve son paradigme dans le récit ${ }^{9}$.

Laissons de côté les analyses de Ricœur pour ne souligner que les présupposés qui le lient à la pensée de Heidegger. Certes, Dilthey n'est pas loin de cette conception qui fait de l'histoire le récit de formes de

6. P. Riceur, Du texte à l'action. Essais d'herméneutique, t. 2, Paris, Seuil, 1986, p. 25.

7. P. Riceeur, Histoire et vérité, Paris, Seuil, 1955.

8. P. Ricceur, Finitude et culpabilité, t. 2, Paris, Seuil, 1960; De l'interprétation. Essai sur Freud, Paris, Seuil, 1965; Le conflit des interprétations, Paris, Seuil, 1969.

9. P. Riccur, La métaphore vive, Paris, Seuil, 1975; Soi-même comme un autre, Paris, Seuil, 1990; Temps et récit, Paris, Seuil, 1984, 1985 et 1986. 
vie. Mais, à la suite de Heidegger, Ricœur lui donne une signification ontologique. On le sait, la compréhension heideggérienne désigne une manière d'être. En mettant le Dasein en relation avec lui-même, elle le constitue comme tel. Rappelons que cette compréhension, toujours située, par laquelle le Dasein investit l'étant et s'investit comme étant dans l'être, fonde sa capacité au discours (Rede). On ne saurait isoler la compréhension du discours, qui désigne ici une manière d'être et qui fonde toute écriture herméneutique. On peut dès lors inférer que la narrativité n'a pas seulement une signification épistémologique, celle qui consiste à configurer le temps par le récit ni une signification ontique, celle qui consiste à refigurer le temps ainsi configuré par une histoire déterminée. Il existe une narrativité ontologique qui lie la re-citation de soi à la constitution de soi, aussi bien individuelle que sociale. Ce sont ces structures qui nous autorisent à dire qu'il y a une auto-constitution de soi comme sujet narratif qui se vérifie dans les mises en œuvre des récits. Or, n'est-ce pas cette narrativité ontologique qui se trouve fondée dans le discours (Rede) de Heidegger, dans la conscience discursive que Gadamer définit comme une capacité d'énonciation et, d'une façon plus radicale encore, dans l'inconscient narratif de la psychanalyse?

\subsection{La psychanalyse}

Le travail de Ricœur sur l'œuvre de Sigmund Freud vérifie l'idée selon laquelle la psychanalyse, entendue comme discours et comme pratique, est une herméneutique. Si nous partons de la situation analytique, nous observons, en effet, qu'elle se présente comme une situation de langage. Mais cette situation reste particulière, car le dialogue qu'elle autorise se déroule dans une ouverture insolite, aménagée par le transfert. Elle ne se réduit pas à un processus empirique de type communicationnel, car elle en appelle à un champ qui n'est plus celui du dialogue intersubjectif. Le transfert est la scène où les événements du passé du patient se trouvent rejoués ou, plus précisément, re-traduits et attribués, sur le mode de la fiction, au désir de l'analyste. C'est cette traduction que l'analyste doit assurer et refuser à la fois, pour ouvrir au patient la seule issue possible: celle d'une interprétation qui inflige un démenti au passé, pour que ce dernier puisse être ré-écrit sous l'horizon d'un avenir prometteur. Le transfert reste le milieu incontournable où s'inscrit la situation de parole: le dire y devient adire et errance, il s'y dissémine, la parole s'y délie et y 
trouve écho. Le locuteur, arraché à la position duelle d'émetteur et de récepteur, s'y retrouve comme témoin de ce qu'il dit. Pour reprendre le modèle de Gadamer, le transfert a pour objectif de libérer «les virtualités langagières »: l'énonciation annonce (hermèneuei), sous le masque des énoncés interminables, le désir du sujet. C'est l'écoute du désir par l'analyste qui empêche le blocage du transfert dans les procédures imaginaires d'une traduction définitive et qui rend cette annonce possible.

On peut se demander si cette position du transfert qui caractérise la relation analytique n'est pas la résultante d'une théorisation spécifique de ce qu'implique toute situation herméneutique. Il n'y a pas, en effet, d'herméneutique possible sans qu'un champ de dialogue ne soit au préalable ouvert. Telle est la fonction de précompréhension des préjugés, au sens où l'entend Ricœur. Point de rencontre avec autrui, avec l'œuvre ou avec le texte, sans attente, sans empathie, sans anticipation. L'ensemble de ces attendus constitue, semble-t-il, le cercle herméneutique, injustement décrié parce qu'on ne comprend pas qu'il puisse être la délimitation du champ préalable à tout travail d'interprétation. Voici pourquoi nous sommes tenté de voir, dans le transfert, un dispositif analogue au cercle herméneutique, mais plus élaboré et obéissant à des règles précises. La métaphore de la circularité n'est pas erronée: la parole qui annonce le désir du sujet, parce qu'elle s'énonce "du » transfert, épouse, en effet, un tracé circulaire, mais elle peut, à tout moment, soit tourner en rond sur une trajectoire fermée, soit évoluer, de façon ouverte, en suivant le tracé de la spirale. Ainsi, le transfert, qui reste un concept élaboré par la psychanalyse, se trouve convoqué, sous des formes plus larges, dès que la compréhension obéit à l'interprétation.

Ceci étant posé, on peut considérer que la psychanalyse traverse, à sa façon, les carrefours connus de l'herméneutique: ceux qui vont de la compréhension à la levée de l'oubli des fondements, en passant par le dépliement des visées implicites de la conscience. Comme le propose la démarche de la phénoménologie, celle de la psychanalyse entend parcourir le chemin allant de l'apparence à la racine inconsciente de l'être, en effeuillant les diverses couches de l'apparition du sens. Mais la phénoménologie se satisfait de considérer la subjectivité transcendantale comme étant la source même de la constitution du sens. Ce que la psychanalyse, quant à elle, ne saurait admettre: elle met en question le phénomène même de cette conscience dite originaire, en cherchant, en amont de l'intentionnalité thématique, l'intentionnalité en acte qui fonde toute 
phénoménalité. Tout se passe comme si le sens restait à jamais séparé de la conscience qui le pose, comme si la conscience émergeait, comme phénomène, au cœur d'une inscience qui fait le lit de l'inconscient, tel que Freud le postule. Car il s'agit, bien sûr, de part et d'autre, d'un postulat que l'on peut toujours falsifier.

Certes, une telle inscience est bel et bien reconnue et posée par la phénoménologie, dans le retrait de la conscience. Que l'on pense aux postures cinesthésiques de Husserl, à la chair qui, selon Maurice MerleauPonty, donne corps à l'intentionnalité, à l'être-au-monde de Heidegger qui dresse l'arrière-fond de l'intentionnalité. Mais la psychanalyse lui donne un statut herméneutique, dans la mesure où ce sont les affects, les pulsions et le désir qui s'annoncent, à défaut de s'énoncer, dans le champ de la conscience. En radicalisant la rupture qui existe entre l'inconscient et le conscient, la psychanalyse ne peut plus se satisfaire de la réduction phénoménologique: elle recourt, d'emblée, à l'herméneutique comme étant l'unique accès possible au dévoilement de l'inconscient. En d'autres termes, l'interprète-phénoménologue examine le sens en s'appuyant sur la traduction des vécus de conscience d'un jeu de langage à un autre. Le psychanalyste, en revanche, sans pour autant renoncer au parcours phénoménologique de la traduction, procède par interprétation : il s'emploie à écouter l'écho supposé inconscient de la parole proférée par le patient. Au bout du compte, la compréhension herméneutique, approximative, hésitante, trans-codante et questionnante prend le relais de la compréhension traductrice, qui reformule, code et décode le message.

L'herméneutique analytique suppose bien, comme l'indique Ricœur, non une réduction à la conscience, mais une réduction de la conscience ${ }^{10}$. Cette suspension radicale est la condition préalable à l'écoute des représentants des pulsions. On se tromperait cependant à croire que ces derniers, que Jacques Lacan traite comme des signifiants, sont directement accessibles à l'écoute analytique. Ce sont, en réalité, les effets de ces représentations inconscientes qui affleurent la conscience. Or, ces effets, ce sont les affects dont on comprend mieux que Freud ne leur refuse pas l'accès de la conscience. L'herméneutique analytique est donc l'écoute interprétative des affects qui phénoménalisent, pour ainsi dire, les représentations des pulsions.

10. Ricceur, De l'interprétation, p. 412. 
Les deux orientations herméneutiques, celle de la phénoménologie et celle de la psychanalyse, prennent en compte les affects, comme le remarque fort judicieusement Michel Henry ${ }^{11}$, tout en indiquant qu'il y a deux modes hétérogènes de la phénoménologisation de ces affects. Mais il faut ajouter qu'il y a aussi deux modes possibles de traitement herméneutique: par l'intuition intentionnelle et par la saisie réflexive, d'une part, et par l'écoute interprétative, d'autre part. D'un côté, l'on aboutit à l'être-au-monde du Dasein ${ }^{12}$, aux cinesthésies de la conscience, à la chair du monde. D'un autre, l'on débouche sur le paysage de l'inconscient. Ces deux visées sur les fondements de l'être sont certes hétérogènes, mais elles revendiquent bel et bien plusieurs traits communs, parmi lesquels un même statut de résistance, un même mode d'inhérence dans l'être-humain sous forme d'autoaffection ou encore une même émergence dans le langage. En relevant ces isomorphismes, on indique finalement que la logique herméneutique est inséparable des contraintes de la méthode et de la nature du champ.

L'écoute analytique se déroule dans l'espace ouvert par le cercle herméneutique insolite que matérialise le transfert en acte. Le patient narre les multiples récits qui composent, à ses yeux, le profil de l'histoire de sa vie. L'analyste, quant à lui, opère, grâce à l'écoute flottante qu'il pratique, un tri parmi les éléments énoncés. Il opère une réduction entre ce qu'il juge essentiel et ce qu'il considère comme accessoire. Il déconstruit pour ainsi dire le récit qu'il entend, pour n'en retenir que les traces signifiantes. L'herméneutique est une phénoménologie de l'écoute. Elle s'accomplit à lire le texte qu'elle écrit. Texte insolite constitué de nœuds signifiants qui rythment les moments de la citation de soi que voile le flux de la récitation. Voici que l'écoute brise la continuité du discours, en restitue la discontinuité constitutive, pour en produire le texte. Mais il va de soi que le patient collabore lui aussi à cette production. C'est à entendre le texte supposé que l'écoute flottante ne cesse d'écrire que s'accomplit l'analyse. Ainsi distinguera-t-on trois niveaux dans cette mise en œuvre de l'interprétation: celui de la constitution de la précompréhension réalisée dans le cercle herméneutique transférentiel, celui de l'écriture herméneutique du texte et celui de l'appropriation herméneutique de ce dernier.

11. Henry, «Ricœur et Freud», p. 141.

12. On peut déceler, à suivre Heidegger (voir Vom Wesen des Grundes), un processus d'auto-affection dans la genèse de l'être-au-monde: l'ipséité résulte de cet «affect » radical. 
C'est, on le sait, Lacan qui pose les bases épistémologiques de cette démarche à partir d'un double principe: celui de «l'inconscient structuré comme un langage » et celui du "signifiant qui représente le sujet pour un autre signifiant». L'analyse déchiffre les traces de l'inconscient que sont les signifiants. L'interprétation se passe ici de commentaire, c'est-àdire de toute traduction. C'est le sujet, glissant sous la chaîne signifiante qu'il convient de surprendre dans les effets d'annonce des signifiants. Point de réalité à restituer, point de traduction à faire d'une vérité précodée. L'herméneutique a certes pour objectif d'instruire le texte qui s'écrit au fil du récit produit et entendu dans le transfert. Mais elle va bien au-delà, puisqu'elle cherche à atteindre la Lettre, c'est-à-dire les structures ou les postures fondamentales de la constitution du sujet. Entendons, par là, les points d'articulation du désir à l'écriture, les points d'articulation de l'écriture au réel, ou, si l'expression ne prêtait point à ambiguiité, les existentiaux «symboliques» du sujet. C'est de la Lettre que la lettre du récit témoigne, c'est de la Lettre que le texte rend compte. Le schéma lacanien est, on le voit, très proche du schéma derridien de la différance, c'est-à-dire du point d'ancrage de la réalité dans l'aleph de l'écrit. À condition, toutefois, de préciser que, selon Lacan, la Lettre désigne l'effraction symbolique du sujet dans le champ du réel. C'est, en effet, cet abord symbolique du réel qui définit l'herméneutique et, à ce titre-là, il s'appuie sur «une revendication herméneutique... qui cherche la signification toujours neuve et jamais épuisée, mais menacée d'être coupée en herbe par celui qui trouve ${ }^{13}{ }^{\prime}$.

\subsection{Théologie et religion}

Le modèle de l'herméneutique religieuse recouvre, à l'origine, celui de l'exégèse, qui désigne la méthode d'interprétation des textes bibliques. Il repose sur la théorie bien connue des quatre sens de l'Écriture: le sens littéral qui s'attache à la lettre, le sens spirituel ou allégorique qui dégage les correspondances entre l'Ancien et le Nouveau Testament ou entre l'Écriture et le dogme, le sens moral ou tropologique qui pose les règles de la conformité de l'action à l'Évangile, enfin, le sens anagogique ou mystique qui initie le lecteur aux secrets de l'Esprit. Mais l'exégèse

13. J. LACAN, Les quatre concepts fondamentaux de la psychanalyse. Le séminaire, livre XI (1964), Paris, Seuil, 1973, p. 12. 
ramène ces quatre sens à un seul: elle reste, en effet, tributaire d'une conception "dogmatique» de la vérité et du texte. Elle présuppose qu'il y ait, entre ces différents sens, un accord a priori qui interdit toute dérive de l'interprétation. Le sens mystique lui-même ne saurait s'imposer au point de briser l'équilibre d'un tel accord qui concourt à asseoir l'intelligence de la vérité de la foi. On le voit, l'interprétation exégétique s'appuie sur des codes littéraires, symboliques, pratiques et fiduciaires, pour traduire le message de la Révélation. Elle reste soumise à la vérité supposée d'un texte qui fournit à l'interprète les instruments techniques dont il a besoin, sans que ce dernier ne soit pour autant obligé de recourir à des instruments extérieurs, à ceux d'une philosophie qui échapperait à la tutelle de la religion. L'exégèse se refuse à interroger le statut épistémologique de la parole de Dieu et à accorder à l'interprète une part active dans la restitution du message. C'est l'herméneutique qui assure cette tâche, en recourant à des modèles d'interprétation qui ne relèvent pas des seules disciplines relatives à l'intelligence du texte, comme la grammaire, la logique et la rhétorique, mais à d'autres disciplines comme la philosophie, l'anthropologie et les sciences humaines. L'herméneutique supplante l'exégèse dès que l'interprète sort du champ du texte pour prendre en compte le vécu du croyant et dès qu'il recourt, pour ce faire, à des règles ou à des repères qui appartiennent à son équipement culturel. Il s'agit, en quelque sorte, de traduire le message religieux dans un langage compréhensible, pour qu'il puisse être signifiant et intégré à la visée du croyant.

\subsubsection{Bultmann}

Cette démarche se trouve illustrée, de façon éclairante, par la démarche de Rudolf Bultmann, qui a tenté d'éclairer la pertinence du message chrétien en s'aidant des catégories de l'analytique existentiale de Heidegger. Elle est un modèle du genre, dans la mesure où elle met en dialogue les contenus dévoilés par l'exégèse avec les attentes fondamentales de l'existence humaine. Un tel rapprochement n'a, aux yeux de Bultmann, rien de choquant, si l'on considère que le Dieu de la Révélation est aussi bien le Dieu créateur de l'être humain. Rien d'étonnant, dès lors, à ce que la compréhension de la Parole de Dieu trouve, dans l'existence humaine, des "points d'attache» ou des structures déterminant la précompréhension du message. 
L'herméneutique de Bultmann vise à rendre intelligible la démarche de foi. Croire, c'est acquiescer au mystère de la croix et de la résurrection du Christ. Ce qui suppose, d'une part, que le croyant renonce au mensonge de l'existence inauthentique et, d'autre part, qu'il libère les possibilités de l'existence authentique. Mais le message chrétien est enveloppé dans le langage du mythe qui l'objective et tend, finalement, à occulter la merveille de l'événement (Wunder) sous le revêtement merveilleux (Mirakel) du récit. On comprend dès lors que la décision de foi se réalise en deux temps: celui, préalable, de la démythologisation qui déconstruit le mythe et celui, essentiel, de l'adhésion à la Révélation. L'herméneutique de Bultmann recourt à l'analytique existentiale pour interpréter l'acte de foi: croire, c'est comprendre ses propres possibilités et les mobiliser par une décision radicale. D'un côté, elle relit, à la lumière de la philosophie de Heidegger, le contenu du message chrétien, en vue de montrer qu'il accomplit les attendus de l'existence. Mais, d'un autre côté, elle prolonge ces attendus, en leur appliquant un contenu "existentiel» pour le moins inattendu et, surtout, en corrigeant les structures existentiales elles-mêmes, puisque la "résolution" à la mort se convertit en espoir de résurrection. Une telle correction est pour le moins étonnante, lorsque l'on sait par ailleurs la fonction herméneutique radicale que joue, chez Heidegger, l'être-pour-la-mort.

Nous avons là un modèle éclairant de la trajectoire que suit l'herméneutique dans le domaine religieux. La revendication herméneutique se prolonge en subversion herméneutique. Sans ces remaniements, le mouvement de l'interprétation se trouve réduit à une réflexion transcendantale qui, sans modifier ses présupposés, définit a priori les conditions d'accueil du phénomène. Interpréter consiste à faire le tri entre les données de base de la réception du message, à distinguer, comme nous y invite Gadamer, les préjugés ambigus des présupposés féconds et pertinents. On se tromperait à déclarer illégitime cette entreprise qui transforme les données de base de l'interprétation. Il n'y a pas d'herméneutique qui n'en vienne à contester le point de départ de sa trajectoire. On ne saurait faire le grief à Bultmann d'avoir, sur un point essentiel, dit non à Heidegger, pour légitimer le "oui » à Dieu. L'interprète ne craint pas de faire violence à l'épistémologie. 


\subsubsection{Derrida}

Le modèle bultmannien subordonne l'exégèse à une herméneutique interne à l'expérience chrétienne. Mais on pourrait adopter, avec Derrida, une tout autre perspective qui, d'un point de vue externe, s'emploie à déconstruire l'idée de Révélation commune aux religions du Livre. Une telle entreprise ne se limite pas au projet de la généalogie de la morale dont se nourrit, selon Friedrich Nietzsche, la religion, ni à l'opération d'un retournement, le retournement herméneutique qui révèle l'essence du christianisme, comme le propose Ludwig Feuerbach. Il prend la religion comme un fait dont la déconstruction s'emploie, d'abord, à restituer la provenance étymologique des termes-clefs, ensuite à analyser le champ historique, anthropologique et institutionnel où se produisent les filiations et les généalogies historico-sémantiques de la religion, enfin, à dégager les effets pragmatiques qui soustraient peu à peu les mots et les significations à toute mémoire archaïque ou origine supposée.

Le premier volet de ce programme herméneutique nous renvoie aux deux sources étymologiques du mot religio: celle de la tradition cicéronienne (relegere: cueillir, assembler) et celle de la tradition tertullienne (religare, lier, relier). Ces deux sources sémantiques se recoupent, semblet-il, au lieu d'une expérience qui implique une double posture: celle de l'attention scrupuleuse et du retrait sacral qui exige recueillement, réticence, renoncement (re-legere); celle du lien qui, inventée par les chrétiens selon Émile Benveniste, nous met face à l'obligation, au devoir, à la dette. Le deuxième volet porte sur le champ qui s'ouvre au carrefour de ces deux visées: l'une constituée par le foyer de la sacralité, de la sainteté ou de l'indemne et l'autre, par le foyer de la croyance tissée par le lien de crédit accordé à la bonne foi du tout autre ${ }^{14}$. C'est ce double foyer sacral et fiduciaire qui délimite le champ où foi et savoir se croisent au lieu de la raison.

Loin d'opposer, comme Heidegger, croyance et pensée, Derrida, fidèle à la tradition des Lumières, souligne l'engagement de la croyance qui se trouve, selon lui, exprimé par l'acquiescement ( $Z u$-sage) heideggérien de la pensée à elle-même. Au fond de la croyance comme de la pensée, il y a attestation, adhésion, réponse, responsabilité. Le sens de

14. J. Derrida, Foi et savoir, Paris, Seuil, 1996, p. 54. 
la religion est inhérent au langage qui est réponse (res-pondere) à l'appel d'une promesse (spondere), attestation, témoignage. Enfin, le troisième volet d'une herméneutique de la religion doit prendre en compte les relectures politiques de ces postures de base. Ainsi le foyer sacral de l'indemne est-il livré à un double destin: celui de protester de sa pureté, de se protéger contre sa propre protection, de s'insulariser ou de rallier le cœur du désert; celui, opposé, d'affronter le danger extérieur, de s'en servir comme d'une médiation, pour s'affirmer, sans pour autant renier son enceinte identitaire ${ }^{15}$. Quant au foyer de la croyance, il suit une trajectoire analogue à celle de l'indemne: soit en revendiquant le respect d'une loi particulière, celle de la culture religieuse, soit en ralliant la loi universelle de la raison qui, selon Kant, est le lieu d'une foi réfléchissante. On le conçoit, l'islam a épousé la première trajectoire. En revanche, le christianisme, en raison même de son message qui met Dieu en abyme aussi bien dans le symbole de la croix que dans le symbole trinitaire, a suivi la seconde trajectoire. Le parcours culturel qu'il a effectué, d'Athènes à Byzance, en passant par Rome et Jérusalem, l'a préparé à s'allier à la démocratie et au progrès machinique et technique qu'il a luimême contribué à produire.

La démarche herméneutique de Derrida privilégie les religions du Livre centrées sur l'événement d'une Révélation. C'est à questionner cet événement fondateur qu'elle accomplit sa tâche. D'une certaine façon, la Révélation est la différance ou, en termes lacaniens, la Lettre constitutive de la religion. Il existe une manière hégélienne de l'interpréter comme discours de l'Absolu, qui la dissout dans l'onto-théologie. Il existe aussi une manière heideggérienne de l'interpréter comme ouverture, qui la protège "scrupuleusement". C'est sur le statut même de cette ouverture qu'il reste à s'interroger. Dans Sein und Zeit, celle-ci est pensée sur le mode luthérien de l'appel de la conscience (Gewissen), appel (Ruf) qui prend la forme de la dette ou de la faute et qui est la transcription ontologique de la «lumière naturelle» (lumen naturale) qui, selon la philosophie scolastique, définit la capacité de l'homme au surnaturel. Mais dans les écrits postérieurs, l'appel de la conscience se transforme en révélabilité (Offenbarkeit), concept qui nous fait passer du registre sémantique de la dette à celui du don. Reste pourtant à savoir quelle est

15. Derrida, Foi et savoir, p. 69. 
la relation existant entre l'événement religieux de la Révélation et la Révélation conçue comme Révélabilité. Est-ce l'idée de Révélabilité qui a fourni le modèle herméneutique pour poser celui de la Révélation religieuse? N'est-ce pas plutôt le thème de Révélation religieuse qui a servi de décalque au schème de Révélabilité ? Cette question qui reste en suspens est bien la question de la question: celle qui pointe l'énigme de l'homme à laquelle la religion tente d'apporter une réponse ${ }^{16}$.

\section{Carrefours}

Il existe, entre les divers parcours précédents, un ensemble de lieux communs qui définissent les traits constitutifs de la logique herméneutique. Il nous semble, d'abord, que cette logique formalise une démarche spécifique du discours philosophique comme tel. La philosophie, et cela dès ses débuts avec Aristote, compare les avancées de la pensée à une marche qui se fraie un chemin au travers de thèses hétérogènes, sinon contradictoires. Or, cette marche consiste à "traverser " (diaporein) ces thèses pour en restituer le sens à la lumière des questions posées. Ainsi, c'est le questionnement présidant à cette quête qui déploie l'horizon de la reprise interprétative des théories étudiées. Le philosophe obéit à une méthode qui allie argumentation et démonstration. L'argumentation débouche sur une compréhension que la démonstration transforme en explication. On le voit, le procès de l'interprétation philosophique conjugue compréhension et explication. La première, qui relève de la rhétorique, ouvre à l'intelligence des opinions, des idées et des théories; la seconde, qui fait appel aux ressources de la logique, légitime et valide objectivement les données préalablement comprises.

Quel est l'aboutissement d'une telle démarche? La réponse d'Aristote se dédouble, mais en apparence seulement. La marche est heureuse (euporein) quand elle débouche sur une belle saisie de la vérité. Elle est malheureuse (aporein) quand elle rate son but et déboîte sur les chemins perdus de l'aporie. Mais, fidèle aux exigences de son discours, le philosophe se demande ce qui s'annonce (hermèneuei) aussi bien dans cette brusque clarté de l'horizon qui se lève que dans la redoutable tombée du brouillard de la nescience. Il ne saurait pour autant se réjouir trop tôt ou

16. Derrida, Foi et savoir, p. 28. 
bien encore s'attrister sans raison en renonçant à l'ironie. C'est l'ironie herméneutique, consubstantielle à la philosophie, qui libère aussi bien le questionnement prématurément retenu dans la réponse que la réponse prématurément différée dans l'aporie. Voilà finalement l'idéal-type du parcours herméneutique, tel qu'il commande le paradigme de la rationalité philosophique. C'est bien cette même trame qui sous-tend les modèles dont se réclament la phénoménologie, la psychanalyse et la théologie.

Le champ de l'herméneutique est celui de l'écriture, qui revêt ici deux formes principales: celle, codée, du texte ou des inscriptions vécues et celle, parlée, du dialogue philosophique ou analytique. L'interprétation est, par conséquent, une lecture qui se met à l'écoute du texte ou une écoute qui s'emploie à lire le sens des paroles échangées. La lecture est une écoute de l'écriture et l'écoute est une lecture des effets d'écriture de la parole vive. Ainsi, qu'il s'agisse du texte, du monde, du vécu, d'autrui ou de Dieu, l'herméneutique suppose que l'objet à interpréter soit au préalable réduit à l'écriture d'un langage ou au langage d'une écriture. Un tel présupposé est sans doute partagé par les trois orientations que nous venons d'analyser: la phénoménologie étudie les inscriptions stratifiées qui sont inhérentes aussi bien au phénomène observé qu'aux visées de la conscience; la psychanalyse étudie l'écriture de l'inconscient structuré comme un langage et la théologie l'écriture de la parole de Dieu, au sens à la fois subjectif et objectif du génitif. Mais cette décision épistémologique n'est pas le fruit d'une généralisation postérieure à la charte méthodologique. Schleiermacher définit les bases d'un art de comprendre qui déborde la visée étroite de l'exégèse et inclut la psychologie de l'individu engagé dans un style d'écriture. Dilthey, quant à lui, donne à cette conception une assise plus large, en montrant que les formes du langage sont inséparables des formes de vie.

Il est un autre lieu commun au geste herméneutique. Il découle de la manière dont le travail de l'interprétation aborde l'objet. Le texte ou le discours présente, en effet, deux versants: l'un littéral, l'autre figuré ou encore l'un manifeste et l'autre caché. Mais on se tromperait aussi bien à concevoir que le premier sens reste un invariant, enchaîné qu'il est à la lettre, tandis que le second sens reste mouvant, emporté qu'il est par l'esprit qui le recrée sans trêve. Comme s'il y avait, d'un côté, l'objectivité de la lettre et, de l'autre, la vérité de l'esprit. Mais l'illusion inverse est tout aussi tenace. Elle consiste à revendiquer le modèle d'une double vérité, littérale et spirituelle. On s'en doute pourtant: c'est en déconstruisant la 
lettre que l'herméneutique libère le sens caché. Du même coup, c'est la lettre qui se transforme en s'inscrivant dans une visée inédite de significations impensées ou, plus justement, c'est l'esprit qui se cristallise dans une lettre nouvelle qui sert désormais d'appoint ou de point de départ à d'autres tentatives d'interprétation.

On le voit, le corps de la lettre n'est pas la Lettre qui, chez Lacan, pointe, à l'instar de la différance de Derrida, l'ancrage de l'écriture dans le réel. Il délimite et circonscrit le sens qui se dépose à même cette lettre résistante que l'interprétation déporte et déplace. On comprend dès lors qu'il n'y a pas de sens qui soit imposé par la lettre ou érigé en vérité ultime par l'esprit. Disons qu'il n'y a point de vérité absolue, échouant dans un sens ultime, susceptible d'être aussi bien contenue dans la lettre que retenue au nom de l'esprit. L'illusion du sens littéral provient de la confiscation par l'institution d'une codification provisoire du sens. La vérité, quant à elle, se situe entre la lettre et son Autre, qui est la Lettre. Elle est la différance, qui s'abîme, à peine surgie du travail de lecture qui ne cesse de lier et délier le prétendu sens littéral.

Observons que la quête de fondement n'a pas le même objectif, selon que l'on ait à faire avec une démarche philosophique ou avec une démarche psychanalytique. La réflexion philosophique et le travail de l'analyse visent, chacun à leur façon, la vérité, mais quel que soit le clivage herméneutique, la première recherche celle-ci sous forme d'un fondement objectif (Grund) fourni par l'idée de Dieu, par le sens codé du texte, par les «ipsissima verba", par l'écriture du monde et des choses, par l'ultime strate du vécu... La philosophie, on le voit, n'a pas coupé avec la logique philologique en mal d'origine. Généalogie nietzschéenne, destruction heideggérienne, déconstruction derridienne: autant de gestes revendiquant la vérité d'une lettre qui insiste et résiste, d'une lettre qui joue à l'être, d'un sens qui investit soudain un espace disponible ou, plus justement, s'investit lui-même en espace dispos. L'idée de fondement surgit dès que le littéral se fait littoral: terre promise où l'on croit aboutir, île où l'on se réfugie, désert d'où l'on désensable les hiéroglyphes. La raison philosophique redouble le logos qu'elle est pourtant: elle fige la parole à la caresser. Verweile dich, $d u$ bist so schön. À quelles fins?, questionnera-t-on. À des fins de partage. La raison est relation: elle saisit, en instantané, le bon moment, pour en faire les assises du partage. L'herméneutique réglemente un tel partage. 
Le motif le plus insistant de l'herméneutique est, sans nul doute, celui de la compréhension. Ce dernier, à la différence de l'explication, qui recherche les lois d'un phénomène donné par déduction ou par «induction logique », procède par «induction artistique », selon les termes de Hermann von Helmholtz, c'est-à-dire par intuition, par «réduction », par lecture sur indices, par empathie, par reprise réflexive, par lecture transférentielle... Autant d'approches qui caractérisent la compréhension inhérente à l'interprétation. On pourrait les classer en deux grandes catégories. L'une convoque les procédures de la traduction qui, à partir de règles, essaie de restituer un sens déjà donné ou codé, mais caché et non explicité. Le modèle en est, par exemple, l'interprétation des rêves de Freud, qui cherche à traduire les images oniriques en un langage compréhensible; ou encore, l'interprétation des mythes de Claude Lévi-Strauss, qui restitue la logique rationnelle cachée sous le revêtement imaginaire du récit. L'autre illustre le processus de l'interprétation proprement dite, qui fait essentiellement appel à la lecture intuitive, à l'écoute "flottante", à la réduction eidétique ou encore à la construction d'hypothèses analytiques visant à rendre compte des jeux de langage. D'un côté, le sens est traduit, décodé et recodé; d'un autre, il est lu, entendu, fictionné, déchiffré et décrypté. On le voit, la compréhension fait appel à deux logiques distinctes qui souvent se recoupent: l'une relève d'une interprétation par traduction et obéit à des codes, l'autre d'une interprétation par «transduction" ou par extraction qui recueille les effets de sens lus et entendus.

À juste titre, comme l'a montré Ricœur, le récit est consubstantiel à la compréhension herméneutique, quelle que soit la forme dont elle use. Le rapport privilégié que l'herméneutique entretient avec le récit tient sans nul doute aux pôles autour desquels elle s'est installée: celui de la vie ou du vécu dont la phénoménologie analyse la narration, celui de l'inconscient dont la psychanalyse explore la re-citation, celui de la foi en la parole d'un Dieu qui se révèle dans le récit. Mais il existe une raison plus profonde que Ricœur a remarquablement saisie: elle tient à la configuration temporelle de la vérité que seul le récit peut réaliser. C’est toujours dans un récit explicite ou implicite que se dévoile et se voile la vérité. Heidegger a eu le génie de voir dans la métaphysique le récit polémique de l'oubli de l'être. C'est bien le rapport de la vérité au temps ou encore l'expérience de la vérité comme événement qui, parce qu'il nous «intrigue », en appelle à la mise en intrigue que permet le récit. Être des lointains, l'homme ne peut que recourir au langage de la finitude et 
re-citer l'événement de son être-au-monde. L'identité narrative de l'homme se décline dans le récit, qui énonce le deuil de l'origine abyssale et annonce que tout peut commencer dans l'avenir du présent. C'est à relier, dans la matrice du récit, les traces du passé, les attentes d'aujourd'hui et les exigences de demain que s'emploie l'herméneutique. On comprend dès lors mieux pourquoi la compréhension se réalise, selon M. Heidegger, dans un mode d'être discursif et, selon P. Ricœur, dans une conscience narratologique ${ }^{17}$.

Venons-en au profil du sujet-interprète qui ne peut que revendiquer un rapport problématique à une vérité qui se voile en se dévoilant. Il ne saurait en effet aboutir, en deçà du texte, des visées de la conscience ou de l'inconscient, à la terre ferme de la certitude. Non seulement parce que tout fondement, quel qu'il soit, est susceptible d'être ébranlé par le travail de l'interprétation, mais aussi et surtout parce que la vérité de la compréhension qui donne sens aux données qu'elle saisit n'est jamais accomplie comme telle. Il est, en effet, toujours possible de contester les acquis du passé à la lumière du nouvel horizon qui les dévoile autrement. C'est le telos de l'herméneutique qui découvre la vérité de l'archê qui se donne à déchiffrer ou à décrypter. D'une certaine façon, le parcours de l'interprétation est rétrograde.

On comprend dès lors que le sujet-interprète est toujours en déprise par rapport à la vérité obscure du passé et par rapport à celle, incertaine, de l'avenir. Il n'a aucun absolu auquel s'adosser, aucun absolu auquel mesurer l'horizon d'une vérité livrée au dévoilement de l'histoire et de son histoire. C'est peut-être le sujet de la théologie qui éprouve le plus douloureusement cette déprise ou cette dépossession. Étant eschatologique, la vérité du salut se passe à la limite de tout horizon messianique, comme l'a indiqué Bultmann, puisqu'elle surgit de la décision de foi qui, se nourrissant de la promesse, mène à bon terme la compréhension. Au dire de Heidegger, la vérité est éprouvée dans le comportement ouvert de

17. La théorie du schématisme kantien fonde l'herméneutique, dans la mesure où la finitude humaine nous impose de mettre en images notre rapport aux concepts et aux idées. Schématiser, c'est en appeler au récit pour accéder au discours. On perçoit mieux les conséquences herméneutiques de cette théorie si l'on distingue, avec Heidegger, deux types de schèmes chez Kant: le schème comme "image " du concept et le schème conçu comme image du temps à l'œuvre. Or, ce dernier schème « ontologique » se trouve au fondement du premier schème «ontique » qui se trouve assimilé à l'image. 
l'homme-interprète. Mais ce "comportement ouvert » est lui-même fondé sur la liberté, qui n'est autre que la volonté de légitimer l'existant que l'homme dépasse par la compréhension qu'il en a, mais qui, en même temps, l'investit de toutes parts. L'interprétation consiste à fonder l'êtreau-monde sur le mode interrogatif de ce que Heidegger appelle la motivation. Elle consiste à justifier, subjectivement et objectivement, le pari et le parti pris que prend le sujet à l'endroit de la vérité, en lui proposant des repères pour s'orienter dans le monde.

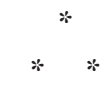

L'herméneutique est née d'une double dérive: dérive de la métaphysique vers la phénoménologie et dérive de la phénoménologie vers l'interprétation... La phénoménologie modélise, en effet, le parcours herméneutique déjà familier du discours philosophique. Mais elle ne va pas jusqu'au bout de ses visées, préoccupée qu'elle est d'adosser, non sans coupable hésitation, la logique de sa démarche à l'absolu de la conscience transcendantale. Mais cette hargne épistémologique, dernier reste d'une culpabilité métaphysique, fragilise l'intentionnalité qui se trouve thématisée sur un déni. Justement, à trop vouloir l'enraciner dans les cinesthésies, dans la chair du monde, dans l'être-au-monde, elle finit par la mettre en abyme et l'effacer, pour faire place à l'inconscient, à la Révélabilité, à la Révélation... C'est ce glissement incontrôlé d'un fondement déclaré vers un fondement hypothétique qui découvre à la phénoménologie le champ de l'herméneutique qu'elle hantait déjà. Il ne s'agit plus d'effectuer une greffe herméneutique sur la phénoménologie, puisque l'entame du rejeton est déjà là, visible et disponible. À défaut de fondement, l'espace de l'herméneutique se trouve libéré. Certes, c'est la finitude qui le délimite. Mais la finitude autorise le geste de la transcendance qui se confond avec celui d'une interprétation indéfinie. 


\section{RÉSUMÉ}

Du point de vue de la démarche philosophique, l'a. présente trois trajectoires herméneutiques distinctes, pour en déterminer les points d'articulation et les convergences: la phénoménologie (Husserl, Heidegger, Gadamer, Ricœur), la psychanalyse (Freud, Lacan), la théologie (Bultmann, Derrida). Quelques «lieux communs» de l'herméneutique apparaissent alors: traversée et quête renouvelée d'une compréhension où il n'y a point de vérité absolue, écriture codée du texte ou des inscriptions vécues et écriture parlée du dialogue philosophique ou analytique, tension entre le littéral et le figuré, compréhension perçue comme traduction et compréhension perçue comme écoute, partage sur la question du fondement, rapport privilégié entre le récit et la configuration temporelle de la vérité, et précarité du sujet-interprète douloureusement éprouvée par le sujet de la théologie.

\section{ABSTRACT}

From a philosophical point of a view, the author presents three distinct hermeneutical trajectories: phenomenology (Husserl, Heidegger, Gadamer, Ricour), psychoanalysis (Freud, Lacan), theology (Bultmann, Derrida). He tries to determine how they are articulated and what are their principal points of contact. Several common areas of the hermeneutical quest then emerge: a renewed quest for understanding where there is no absolute truth; a coded writing of the text or living inscriptions; an oral writing of the philosophical or analytical dialogue; a tension between the literal and the metaphorical; understanding seen as translation and as listening; interest in the question of foundations; the relationship between narrative and the temporal configuration of truth; and the precariousness of the interpreting subject painfully experienced by the subject of theology. 